\title{
Retrospective Analysis of Breast Cancer Cases Operated in Jush within Four Years Time Period, Jimma, Ethiopia
}

\author{
Ruhama Yoseph ${ }^{1}$
}

\author{
${ }^{1}$ A Research Paper Submitted to College of Medical Sciences and Public Health of Jimma University
}

\begin{abstract}
Breast cancer is the second most common cancer in the world. It is also the commonest cancer affecting women with also highest cancer related death rate. However due to the poor cancer registry in developing countries the exact magnitude of the problem is not known. In Ethiopia breast cancer is the second most common cancer next to cervical cancer. The fact that Sub-Saharan African has peculiar epidemiological trends of breast cancer has drawn the attention of many to the region. These peculiar features include relatively younger age of affected women, high mortality rates and triple-negativity of the cancer. Additionally, a poor association with life time estrogen exposure has been observed. Despite the generally observed great burden of breast cancer, Ethiopia is one of the countries where the clinical and pathological characteristics of the cancer are not well researched. This study tries to fill this information gap by studying the situation observed in JUSH, which is the highest referral center available for Southwestern part of the country. The general objective of this study was to retrospectively determine demographic characteristics of patients, risk factors, clinical presentation and pathological description of the breast cancers, the modes of treatment given for breast cancer among cases operated at JUSH from September 11, 2010 to September 10, 2014 GC. The study was conducted in JUSH from August 6-14, 2015 GC and cross-sectional study design was used. The study population was those patients with diagnosis of breast cancer who are electively operated at JUSH from September 11, 2010 to September 10, $2014 \mathrm{GC}$ according to the operation log book in the operating theatre. Data was collected from patients' charts on a structured questionnaire that was filled by the principal investigator. Collected data was cleared, checked and stored. Data analysis was done on computer with the help of SPSS 16.0 program. Through-out the study confidentiality of information was respected. Letters of ethical clearance were obtained from the CBE office of JU before the start of the study. The result of study showed a number of peculiar features which included higher rate of male breast cancer (10.4\%), younger than 50 years distribution of majority of female patients (68\%), the presence of protective gynecologic \& reproductive factors to breast cancer in the majority of the affected women, and late presentation of patients. Majority of patients were having advanced breast cancer (Stage III \& IV disease in 76.8\%) and high percentage of nodal involvement (90.7\%). Despite these findings detailed pathological characterization of the tumor has not been effected and information on receptor positivity status of the cancers is lacking. Additionally, the percentage of patients who pursued adjuvant therapy after surgical management was found to be very low (14.7\%). In conclusion, the epidemiological trend of breast cancer patients seen at JUSH within the mentioned four years' time period was found to have major epidemiological deviations from trends that were seen in the world. The unfortunate late presentation of patients is also manifested by advanced stages of disease at diagnosis that theoretically is beyond curability. Prospective studies focusing on the epidemiology of breast cancer and further characterization of tumor biology \& genetics should be performed in the future. Moreover, clinicians, public health workers and policy makers should work on increasing the awareness towards breast cancer, introduction of breast self-examination techniques and launching of effective breast cancer screening programs.
\end{abstract}

\section{Introduction}

Throughout the years it has been observed that the burden of cancer is increasing worldwide. The most recent report of IARC which was in 2012 has revealed that breast cancer is the second most commonly diagnosed cancer globally with a rate of 1.7 million cases per year next to only lung cancer(6). It has also remained for years as the most frequently diagnosed cancer of women in the world. Since the 2008 estimates, breast cancer incidence has increased by more than $20 \%$, while mortality has increased by $14 \%(5)$. Breast cancer is also the most common cause of cancer related death among women (522 000 deaths in 2012) and the most frequently diagnosed cancer among women in 140 of 184 countries worldwide. It now represents one in four of all cancers in women (7).

However, due to the poor cancer registry system especially in the developing countries lower number 
of cases are reported every year. In the year 2002 it was reported by the IARC that there were 1.15 million new breast cancer cases of which $55.8 \%$ occurred in developed countries and the remaining $44.2 \%$ occurred in developing countries. It was and is still known the burden outweighs in western developed countries though the poor record keeping system in third world countries underestimates the actual number and increasing trend of breast cancer. The relatively higher prevalence in western women can be attributed to their life style predisposing them to the known risk factors such as low parity, early menarche, late menopause, late age at first child birth, use of exogenous estrogen hormones as compared to women in third world countries.

On the contrary, there is a recently arising alarm that breast cancer prevalence is increasing in developing countries. According to statistics report by IARC, the projection for the year 2030, based on the current trend in incidence ratio, reveals that 2.7 million new cases would be diagnosed with more than $60 \%$ of them occurring in less developed countries. This figure is conservatively assuming that the current incidence rates are held constant for all countries which emphasizes that one can predict the burden of breast cancer in developing countries to be increasing in the coming years. This rise in breast cancer cases has also been crudely observed among other SubSaharan African countries. Generally, worldwide trends show that in developing countries going through rapid societal and economic changes, the shift towards lifestyles typical of industrialized countries leads to a rising burden of cancers associated with reproductive, dietary, and hormonal risk factors(2).

It is known that black women living in Africa and those of African descendants living in other countries have peculiar epidemiological trends of breast cancer. These women have been observed to be afflicted at a younger age by breast cancer rather than the common older age group seen in western women. This situation is made worse by high mortality rates from cancer deaths. The pathological character has also been observed to be more aggressive in African women with low positivity seen for estrogen and progesterone receptors and lower expression of the HER2neu gene. Additionally the risks of breast cancer were shown to have poor association with levels of serum estradiol which is contrary to the dogmatic understanding that lifetime estrogen exposure is a crucial factor in the development of breast cancer.

As it is the same in other Sub-Saharan countries breast cancer is the second most common cancer case among women next to only cervical cancer(1). Even though it is generally observed that the flow of breast cancer patients is also increasing in Ethiopia there is information gap in revealing the actual magnitude of the problem. Additionally, the epidemiological trends of breast cancer, characterization of the pathological types, the response to different modalities of treatment and survival analysis have not been well studied in the Ethiopian setups. This study is aiming to fill this information gap by retrospectively assessing the presentations of breast cancer patients surgically operated at JUSH. It focuses on analyzing the common clinical features of patients with breast cancer that come from South western part of the country. And thus the study lays a basis for developing strategies of creating community awareness on breast cancer, for devising ways of early cancer detection and for researching further on pathological characteristics of breast cancer cases in the Southwestern part of Ethiopia.

\section{Background Information}

Jimma University Specialized Hospital (JUSH) is a referral as well as higher medical education training center residing in Jimma town, Oromia Region located around $355 \mathrm{Kms}$ in the South West direction from the Addis Ababa, the capital city of Ethiopia. It was established in 1930 E.C. The hospital has 450 beds and annually 15 million people get inpatient service within its catchment area, which includes populations of Oromia region, SNNP (Southern Nations, Nationalities, Peoples) region and Gambela region. It is estimated that the hospital gives outpatient service for 125,000 people per year. There are three operating theatres used by the department of surgery of JUSH of which one is assigned for trauma and orthopedic cases. The other two tables are used for operating other elective cases for 2 to 3 days per week.

In our hospital (JUSH), there has been a continuous rise in the number of breast cancer patients seen in the surgical department as observed from the monthly morbidity reports by the investigator. As it is common with other African countries, the majority of the patients are observed to come with advanced breast cancer when only palliative therapy could be given to improve the quality of life. However, these are only rough estimates since the actual number of all breast cancer cases (including the inoperable ones) seen in the hospital has not been studied.

Regarding treatment of breast cancer, the setup of our hospital allows us to deliver the respective surgical treatment for breast cancer according to the stage. And sometimes adjuvant endocrine therapy is added. The endocrine therapy given is so far limited to ovarian ablation or Tamoxifen treatment for patients who can afford it. Otherwise, patients who are economically competent are referred to Addis Ababa, to Black Lion Specialized Hospital, to pursue other 
options of adjuvant therapy since oncology unit is yet not established in our hospital.

Having this background, it is the belief of the investigator that further elaboration of breast cancer characteristics and treatment as observed in JUSH can be reflected by the end of this study.

\section{Literature Review}

The pioneer research done in Ethiopia related to breast cancer is the entitled Breast Cancer in an Ethiopian Population, Addis Ababa done by Proffesor Tesema Ersumo. It was a retrospective study that analyzed biopsy proven breast cancer cases seen and surgically treated at Tikur Anbessa Hospital during 1995-99 G.C. Of the total 137 biopsy proven breast cancer cases seen, only the data of 125 could be retrieved and analyzed. The information obtained included the demographic characteristics of patients, clinical \& pathological description of the cancer, the modes of treatment given and outcome of treatment (14).

According to this study, the male to female sex ratio was found to be 1:8 and majority of the females were found to be pre-menopausal. The median parity of females observed was $4 \pm 2$ but still $18.1 \%$ were young and nulliparous. Ethnic group and religion wise most were found to be Amhara and Christians (14).

Regarding the clinical finding on patients, the study revealed that majority $(92.4 \%)$ came with chief complaint of painless breast lump and the median duration of presenting symptom was found to be $11.5 \pm 19.3$ months. About half had locally advanced disease while $25 \%$ had ulcerated and fungating breast lesion at presentation. Pregnancy was also documented on few patients at the time of presentation. History of benign breast disease could be retrieved in only $6 \%$ of patients (14).

The commonest clinical sign of breast cancer seen among the study population was found to be breast mass with axillary metastasis. At presentation, about $54 \%$ had mobile axillary lymph nodes while $16 \%$ had fixed lymphnodes. The upper outer quadrant was found to be the commonest site of where the breast masses were located. And in majority $(60 \%)$ the size of the mass was found to be greater than $5 \mathrm{~cm}$ in diameter. Distant metastasis was seen in $10 \%$ of patients at time of initial presentation and the commonest sites were found to be lung, bone and liver in order of frequency. TNM crude staging was given according to information stated on patients' records. Accordingly, $60.2 \%$ had stage III and stage IV disease. Supraclavicular lymphadenopathy, contralateral lymphadenopathy and satellite skin nodules were observed to be rare. The most frequent pathology report was Ductal Invasive carcinoma
$(77.6 \%)$

The surgical treatments given according to the study included Modified Radical Mastectomy with axillary dissection (71.8\%), Toilet Mastectomy and Breast Conserving Therapy (BCT) with axillary dissection the last one specifically was found to be offered for quite few patients. Additionally, 42 patients underwent combined or single adjuvant therapy which consisted either of radiotherapy, chemotherapy, Tamoxifen and Oopherectomy. Recurrence after treatment was observed to be seen in $45.9 \%$ of cases in the form of local or distant forms (14).

Concerning, the outcome of follow-up, 109 cases $(87.9 \%)$ were found to appear for follow-up for a median duration of 10 months whereas only $58.7 \%$ were found to reappear within a duration of one year. Only four cases were found to continue follow-up for 5 years and more after the initial presentation (14).

This research is pioneer and unique in opening our eyes on the trends of breast cancer among the Ethiopian population. There were also peculiar findings emphasized by the investigator when comparing the findings of the study of breast cancer among Ethiopian patients to that of the world population figures. The significant findings that deviated from the world figures included percentage of nodal metastasis at time of diagnosis, rate of male breast cancer and also proportion of premenopausal and multiparous women affected with breast cancer. The nodal metastasis at diagnosis was found to be $67.2 \%$ in this series which is slightly higher than world figures. The result of this study also showed that the rate of male breast cancer was found to be $10.4 \%$ with a median age of 55 years. The investigator has compared this to the corresponding American figure which is $0.5 \%$ and to findings of African series which ranged from $2.4 \%$ to $15 \%$ (14).

The limitations of this study could be its retrospective nature which made $100 \%$ retrieval of information from patients' records impossible. Additionally, the pathological descriptions of breast cancer cases could not tell more than confirmation of diagnosis and histological type of the disease. It is a general economical shortcoming of Ethiopian setups to fail to determine the standard receptor positivity status and Her-2 neu status from breast cancer specimens and so is this study. Another important factor which one may find difficult to deduct from this study is the survival rates of patients. Although the study has revealed that only few came or reappeared for follow-up, it doesn't clarify the cured or lost cases and their five year survival rates. It only mentions that $4 \%$ have died which obviously would be under the expected figures and shows the difficulty of 
tracing the outcome of the rest of patients.

However, the fact that it showed the majority of Ethiopian women with breast cancer are premenopausal and half are having age less than 50 years is in agreement with other African countries experiences. Excessive late or advanced stage presentations were also found to be remarkable. And so is the rate of nodal metastasis at diagnosis.

Other studies done in Ethiopia have also tried to explain the beliefs and practice around breast cancer. One of those studies was done through interviewing of 69 patients with breast cancer who presented at $\mathrm{TAH}$ at a specified period of time. It assessed the belief, experience and perspective of patients towards breast cancer. From the study the relevant finding that is related with the current study found that early signs and symptoms were ignored frequently and that patients preferred to present to traditional healers. In additions it also revealed that most patients experience stigmatization and social isolation which complicated the discussion and action around breast cancer. From the discussion of this study one can deduct and explain the psychological background behind the commonly seen late presentation of patients despite the presence of health facility close to their living residence(4).

Another study assessing the initial experiences, symptoms and actions of patients in Ethiopia ultimately determined to have breast cancer showed that despite noticing of breast lumps only few were found to seek medical advice within the first one year. Also most participants (69.6\%) ignored symptoms for average of more than one and half years (15).

Proceeding to studies performed in Eastern Africa region on breast cancer, the study conducted in Sudan to investigate clinicopathologic features of breast cancer in Sudanese women is worth mentioning. The study compared he clinicopathologic feature findings with similar studies done in other populations of native Africans, African-American and European origin. It was a hospital based study which obtained data for breast cancer from Radiation and Isotope Centre of Khartoum (RICK) from 2009-2011. Fourhundred ninety-seven (497) women were eligible for inclusion into the study. The study showed majority $(70 \%)$ were 50 years and younger. Majority were found to be diagnosed at late stages (TNM Stage III \& IV) with high tumor grades. Invasive Ductal Carcinoma was found to be the most frequent histology type. And greater than half were found to have nodal involvement at presentation. Estrogen and progesterone receptor positivity status was predominantly found to be negative whereas almost half of patients were found to be HER-2 positive.
Triple negative tumor status was observed in $35.4 \%$. After comparing the findings with that of other populations, the study concluded that breast cancer amongst Sudanese women is characterized by early peak age of onset, advanced stage and more progressive biological characteristics when compared to Caucasian women (11).

In harmony to the Sudanese study, a study conducted in Mali, another Sub-Saharan country, has shown high incidence of triple-negative tumors. This was a prospective study on breast cancer characteristics and risk factors in Malian women seen Bamako University Hospital (10).

This study determined the clinical \& tumor characteristics and known risk factors in 114 patients. The result of the study showed that the median age of patients was found to be 46 years. Most tumors were found to be Invasive Ductal Carcinoma (94\%). According to TNM staging, $\mathrm{T}_{3}-\mathrm{T}_{4}$ was seen in $90 \%$ whereas positive lymph nodes were found in $91 \%$ of cases. Majority (78\%) were found to have grade III cancers. Again majority were found to be both estrogen and progesterone receptors negative the rates being $61 \%$ and $72 \%$ respectively. HER-2 was found to be over-expressed in $18 \%$ of cases. Triplenegativity has a rate of $46 \%$ displaying particularly the aggressive pattern of the disease (10).

The study has finally concluded that there was no significant difference seen in risk factors among Malian women. But, noticeably, the finding of high incidence of triple-negative tumor in Mali and the higher prevalence of pre-menopausal women among the triple-negatives gives additional light on the biological characteristics of breast cancer in SubSaharan Africa (10).

Another study was conducted in Uganda based on the premises that breast cancer epidemiological and tumor behavioral disparities exists between black women in Sub-Saharan Africa and their counter-parts in Western high resource countries. The tried to assess this disparity based on the knowledge that high estradiol exposure is found to be a strong indicator for development of breast cancer. The study design was a case-control study which was conducted in Mulayo hospital, a referral and teaching hospital in Central Uganda. There were 140 female participants half being cases, that is with confirmed histological diagnoses, and half being controls meaning those with normal breasts. Serum specimens were obtained from all participants in addition to clinical information. The result showed that no association was found between level of estradiol and breast cancer which is confirming the hypothesized disparity relative to non-African women in high resource countries. But still the median estrogen 
levels were found o be significantly higher than normal levels in Caucasian women (8).

The study has limitations in that single samples were collected and analyzed. This with other factors may make it sub-optimal for making generalization to the entire country's population. Thus, although it further establishes the observed disparity in breast cancer between Sub-Saharan and Caucasian women, it doesn't explain the cause for this disparity.

A systematic review of breast cancer biology in developing countries including African, Middle East, Eastern Europe, Mexico, the Caribbean and South American was done which assessed breast cancer size, stage, grade, histological type, extra-mammary involvement, hormone receptor status as well as patient demographics on breast cancer patients from the mentioned populations of the world by retrieving information from literatures searched and found to be relevant upto December 2009. Particularly searches done from Africa, researches done in Sudan, Nigeria, Kenya, Tunisia and Tanzania were found to be informative (13).

The study conducted in Sudan which was included in this qualitative review is already discussed in the above paragraphs of literature review (11).

There were three studies reviewed from Kenya and one has revealed that breast cancer is the leading cancer among Nairobi women. The three different studies showed tumor biology as part of each study performed and in about half high grade (grade III) was seen in addition to poor receptor positivity status. One of the three studies showed that $66 \%$ of cases were found to be both estrogen \& progesterone receptor negative (13).

The study that was reviewed form Tanzania was done in a series of 50 women. It revealed that $76 \%$ had stage IIIB disease whilst none had stage I disease. Metastatic disease constituted $10 \%$ of all cases. All tumors were found to be Invasive Ductal Carcinoma. Another study from Tanzania assessed tumor markers in 60 patients and expression of estrogen receptor was found to be $33 \%$ and that of progesterone receptor was found to be $18 \%$ only (13).

In summary, the systematic review of breast cancer biology in developing countries showed that breast cancer in those parts of the world is in general characterized by early peak age of onset and aggressive biological characteristics. Particular emphasis was also given on the higher frequency of triple negative tumors seen among African women than any other mentioned populations (13).

Another study that reviewed publications on breast cancer in Africa from the year 1988 to 2004 was done in USA in order to explain possible genetical transmissions of breast cancer trends seen to AfricanAmerican women. It is a studied fact that AfricanAmerican women have had lower incidence of but higher mortality rater from breast cancer when compared with White-American women. They also have higher risk of early onset, high grade, node positive, and hormone receptor negative diseases. This review was done based on the speculation that risk factors could be genetically transmitted from their African forefathers and thus necessitating study of breast cancer among women of Sub-Saharan Africa because of their shared ancestry (2).

The comparison between epidemiological trends of breast cancer in Sub-Saharan Africa and the trend in industrialized nations has shown the lower incidence of breast cancer, an averagely 10 years younger distribution of age at diagnosis, and the advance disease stage, and higher mortality rates of breast cancer in the prior group (2).

Estimates of age-standardized incidence rates (per 100,000 women) were found to be 20.2 in Eastern Africa,13.5 in Middle Africa, 24.8 in Western Africa and 31.8 in Southern Africa. This finding is in contrary to the age-adjusted breast cancer detection rates of 95-100 cases per 100,000 in North America and other Western countries. This was attributed to the largely protective reproductive history including late menarche, early menopause, high parity with prolonged breast feeding culture, irregular menstrual cycles and fewer ovulatory cycles (2).

Nevertheless, the review indicated that breast cancer rates are rapidly increasing in developing countries as in the increment trend that was observed in Uganda. The study mentions that the incidence of breast cancer in Uganda has doubled from 11 per 100,000 in 1961 to 22 per 100,000 in 1995. But the authors have emphasized that the lack of cancer registry in many of the countries makes it difficult to obtain accurate incidence rates (2).

The other important epidemiological characteristic observed among the Sub-Saharan women is the high likelihood of being premenopausal. Breast cancer incidence was found to peak between age 35- 45 years which is 10-15 years earlier than the peak incidence seen in Western countries. But the authors explained that the truncation of life-expectancy in the African setup precludes the ability to make robust conclusions regarding decreasing risk of postmenopausal breast cancer (2).

The review also showed that despite the 5-10 times higher incidence of breast cancer among the Western population, breast cancer mortality is only 2-3 times 
higher which means approaching equivalence to the incidence in some parts of Africa. The relatively higher mortality rates from breast cancer in the African setup were attributed to different factors including delayed presentation, limited therapeutic modalities, and, perhaps, a predisposition to biologically aggressive tumors (2).

This study like the previous mentioned studies has also detected the commonly observed advanced stage of breast cancer in African countries. Different reviewed retrospective studies showed $70-90 \%$ of African women present with stage III or IV disease. The authors have the conjuncture that clinical stage of many patients may be underestimated because of lack of proper diagnostic facilities. Possibility of inherently more aggressive tumor biology was indicated from findings of Hassan et al., which found that 17 to 21 patients $(81 \%)$ with a brief symptom duration ( three months or less) has stage III or IV breast cancer(2).

Finally, the study has concluded that parallels between African-Americans and Sub-Saharan Africans breast cancer patients are seen. It has also forwarded statistical explanations as well as conjectures on the epidemiological characteristics, risk factors, observed rising incidence and persisting high mortality rates of breast cancer in the SubSaharan African setup (2).

A study was conducted on the survival analysis for female breast cancer based on 302,763 adult cases taken from Surveillance, Epidemiology and End Results (SEER) Program of National Cancer Institute (NCI) of United States of America. The influence of extent of disease, histology, receptor status, and demographic factors on female breast cancer survival was studied. Twelve geographical areas were selected from USA for the analysis of this article which represented $14 \%$ of the population. All registries from the selected geographical areas contributed data for diagnosis years 1988-2001 GC except Los Angeles which contributed data for the years 19922001 GC. Then, the 5 year relative survival analysis was done to estimate the effect of cancer on the survival of the cohort (12).

The results of the study revealed many impressive facts. Among others, it showed that a localized invasive disease of breast cancer has better survival rates as compared to other cancer types. From the demographic profiles, young women were found to have less favorable outcome and relative survival rates increased with age at diagnosis until age 70 . The overall 5-year relative survival rate for blacks was $78 \%$ while it was $90 \%$ for whites. The fact that blacks had less favorable stage at diagnosis couldn't explain the survival differential since within each stage grouping blacks had poorer survival. ER+ and $\mathrm{PR}+$ cancers had 3 year relative survival rates of $97 \%$ compared with only $83 \%$ ER- and PR- cancers. Within each stage, ER status was found to be an important prognostic variable. Even within distant stage five year relative survival rate was $28 \%$ for ER negative cancer while it was much higher $(50 \%)$ for ER positive women. It was also found that survival rates generally decreased as the tumor size and the number of lymph nodes involved increased. Survival curves based on the TNM staging system as per AJCC, $5^{\text {th }}$ edition was also analyzed. For patients of all stages the relative 5-year survival was $100 \%$ for stage 0 and stage I cancer while it went down to $20 \%$ for stage IV cancer. However, there was significant variation is survival within the same TNM grouping (12).

This study has tried to assess the different prognostic factors affecting breast cancer survival. Many of those are confirmatory to finding of other studies. The less favorable survival of blacks in USA remains to be elaborated since their late presentations at diagnosis was not sufficient to explain the survival difference observed between these races.

\section{Objectives \\ General objective:}

The general objective of this study is to retrospectively determine demographic characteristics of patients, risk factors, clinical presentation and pathological description of the breast cancers, the modes of treatment given for breast cancer among cases operated at JUSH from September 11, 2010 to September 10, 2014 GC.

\section{Specific objectives:}

- To determine the prevalence of breast cancer per year from the total number of operated elective cases within the specified period of time

- To describe the socio-demographic trends and possible risk factors of breast cancer among patients seen at JUSH within the specified period of time

- To determine the initial clinical presentation and TNM stage of breast cancer patients seen at JUSH

- To describe the prevalent pathological characteristics of breast cancer cases seen at JUSH within the specified period of time

- To describe the modes of definitive surgical treatment delivered to breast cancer cases seen at JUSH within the specified period of time 
Methodology

4.1 Study Area

The study was conducted at Jimma University Specialized Hospital.

\subsection{Study Period}

The study was conducted from August 6-14, 2015

GC.

\subsection{Study Design}

A retrospective cross-sectional study design was used.

\subsection{Population}

\section{Source Population}

The source population was list of patients who are documented as electively operated at JUSH from September 11, 2010 to September 10, 2014 GC according to the operation log book in the operating theatre.

\section{Study Population}

The study population was those patients with diagnosis of breast cancer who were electively operated at JUSH from September 11, 2010 to September 10, 2014 GC according to the operation log book in the operating theatre.

\subsection{Sampling Technique}

No sampling technique was used as all study units were covered.

\subsection{Measurement}

\subsubsection{Instruments}

Structured questionnaire was prepared based on the specific objectives of the study and identified variables. The structured questionnaire was filled by the principal investigator based on information that is obtained from the operation log book and charts of patients.

\subsubsection{Variables}

\begin{tabular}{|c|c|c|c|}
\hline \multicolumn{2}{|l|}{ Variable } & Theoretically Expected & Actually Available \\
\hline $\begin{array}{l}\text { Independent } \\
\text { Variable }\end{array}$ & $\begin{array}{l}\text { Sociodemographic } \\
\text { Information }\end{array}$ & $\begin{array}{ll}\text { - } & \text { Age } \\
\text { - } & \text { Gender } \\
\text { - } & \text { Residence } \\
\text { - } & \text { Education } \\
\text { - } & \text { Occupation } \\
\text { - } & \text { Ethnicity }\end{array}$ & $\begin{array}{ll}- & \text { Age } \\
- & \text { Gender } \\
- & \text { Residence }\end{array}$ \\
\hline & $\begin{array}{l}\text { Hormonal Risk } \\
\text { factors }\end{array}$ & $\begin{array}{ll}\text { - } & \text { Age at menarche } \\
\text { - } & \text { Age at menopause } \\
\text { - } & \text { Parity } \\
\text { - } & \text { Duration of breast feeding } \\
\text { - } & \text { Age at first child birth } \\
\text { - } & \text { Contraceptive use } \\
\text { - } & \text { Exposure to HRT }\end{array}$ & $\begin{array}{ll}- & \text { Age at menarche } \\
\text { - } & \text { History of breast feeding } \\
\text { - } & \text { Parity } \\
\text { - } & \text { Contraceptive use }\end{array}$ \\
\hline & $\begin{array}{l}\text { Non-hormonal risk } \\
\text { factors }\end{array}$ & $\begin{array}{ll}- & \text { History of previous breast lesion } \\
- & \text { History of alcohol use } \\
- & \text { History of smoking } \\
- & \text { Radiation exposure } \\
- & \text { History of indoor smoke exposure }\end{array}$ & - $\quad$ History of previous breast lesion \\
\hline & Genetic susceptibility & $\begin{array}{ll}\text { - } & \text { Family history of breast cancer } \\
\text { and/or ovarian cancer among first } \\
\text { degree relatives }\end{array}$ & $\begin{array}{l}\text { Family history of breast cancer } \\
\text { and/or ovarian cancer among first } \\
\text { degree relatives }\end{array}$ \\
\hline $\begin{array}{l}\text { Dependent } \\
\text { Variables }\end{array}$ & Clinical Presentation & $\begin{array}{l}\text { Prevalence of breast cancer per year } \\
\text { from total of electively operated } \\
\text { cases } \\
\text { - }\end{array}$ & $\begin{array}{l}\text { Prevalence of breast cancer per year } \\
\text { from total of electively operated } \\
\text { cases } \\
\text { - } \\
\text { Commonest clinical presentation of } \\
\text { study units } \\
\text { Median duration of illness of study } \\
\text { units } \\
\text { TNM stage of cancer at presentation }\end{array}$ \\
\hline & Pathology & $\begin{array}{l}\text { - Commonest histological diagnosis of } \\
\text { study units }\end{array}$ & $\begin{array}{l}\text { - Commonest histological diagnosis of } \\
\text { study units }\end{array}$ \\
\hline & $\begin{array}{l}\text { Treatment \& } \\
\text { Outcome }\end{array}$ & $\begin{array}{ll}\text { - } & \text { Commonest surgical mode of } \\
\text { treatment } \\
\text { - } & \text { Rates of post-operative } \\
\text { complications } \\
\text { - } & \text { Adjuvant treatments given } \\
\text { - } & \text { Prevalence of recurrence after } \\
\text { - } & \text { Surgical treatment } \\
\text { Survival rates per year }\end{array}$ & $\begin{array}{ll}\text { - } & \text { Commonest surgical mode of } \\
\text { treatment } \\
\text { - } \\
\text { Rates of post-operative } \\
\text { complications } \\
\text { - } \quad \text { Adjuvant treatments given } \\
\text { Prevalence of recurrence after } \\
\text { surgical treatment }\end{array}$ \\
\hline
\end{tabular}

\subsection{Data Collection}

Study units were all breast cancer patients who were surgically managed within the specified period of time which were identified from the operation log book. Their charts were collected from the record keeping unit of the hospital. And the structured 
questionnaire was filled by the principal investigator based on information retrieved from patients' charts.

\subsection{Data Processing and Analysis}

The data collected was cleared, stored and checked for completeness on daily basis. Data was fed to computer and analyzed using SPSS program version 16. Final presentation was made with graphs, tables and narratives based on the nature of data. Chi-square and $\mathrm{p}$-value was used to determine association between variables and $\mathrm{p}$-value $<0.05$ was considered significant.

\subsection{Ethical considerations:}

- Permission was obtained from medical director of the hospital, department of surgery and JU.

- Formal letter was delivered to major Operation Room and record keeping unit of JUSH.

- The confidentiality of information was respected and results of the study will be disseminated to concerned bodies only.

\subsection{Data Quality Assurance:}

- Cautious matching of information on operation $\log$ book to patients' charts was done.

- The collected data was checked for accuracy and completeness on daily basis.
- Any inconsistent data was rechecked before data analysis.

\subsection{Operational definitions:}

Stages of breast cancer: Based on TNM clinical staging (Source: AJCC cancer staging manual, $6^{\text {th }}$ ed. New York: Springer, 2002, p 227-228)

Advanced breast cancer: Cancer that is Stage III and above

Early Menarche: Menarche earlier than 12 years

Late menarche: Menarche later than 14 years

Early menopause: Menopause earlier than 40 years

Late menopause: Menopause later than 50 years

\section{Results}

According to the objective of the study patients who were operated for breast cancer within the time period September 11, 2010 to September 10, 2014 GC were identified from the operation log book. The time period of retrospective analysis has been reduced from five to four years due to the absence patients' records in the card room who came earlier than five years from the time of the study. Within the mentioned four years' time, there have been a total of 116 breast cancer cases operated at JUSH. Breast cancer was found to be one of the less commonly operated elective cases. The yearly prevalence of operated breast cancer cases is shown in Table 1. The average number of breast cancer cases operated per year was found to be 29 which is significantly lower than the average elective cases operated per year (894).

Table 1: The yearly prevalence of breast cancer cases electively operated at JUSH from September 11, 2010 to September 10, 2014 GC

\begin{tabular}{|l|l|l|l|}
\hline Year & $\begin{array}{l}\text { Operated breast cancer cases per } \\
\text { year }\end{array}$ & $\begin{array}{l}\text { Total electively operated cases } \\
\text { per year }\end{array}$ & Breast cancer prevalence per year \\
\hline $2003 \mathrm{EC}$ & 25 & 796 & $3.1 \%$ \\
\hline $2004 \mathrm{EC}$ & 32 & 825 & $3.9 \%$ \\
\hline $2005 \mathrm{EC}$ & 25 & 888 & $2.8 \%$ \\
\hline $2006 \mathrm{EC}$ & 34 & 1068 & $3.2 \%$ \\
\hline Total & 116 & 3577 & $3.2 \%$ \\
\hline
\end{tabular}

Out of the total 116 cases, the clinical data could be retrieved for 108 cases. But still complete records were barely available making it difficult to withdraw genuine results from some parts such as clinical presentation and risk assessment.

\subsection{Sociodemographic Information}

From the total 116 cases, 103 were female and 13 were male making the rate of male breast cancer $11.2 \%$. The sex ratio was found to be 1:8. Majority of patients were found to be 50 years and above in both sexes $(37.1 \%)$. The youngest age documented was found to be 20 years while the oldest being 85 where both were seen in female patients. The median age at presentation was found to be $45 \pm 13.7$ years.

Among females, $32 \%$ were found to be 50 years and above being followed by the group which consisted of age 35-39 years (18.4\%). Women younger than 30 years age constituted only for $10.7 \%$. Among males majority $(76.9 \%)$ were also found to be 50 years and above. 
Table2: Age by sex distribution of patients with breast cancer who were electively operated at JUSH from September 11,2010 to September 10 , 2014 GC.

\begin{tabular}{|l|l|l|l|}
\hline Age of patients & Female & Male & Total \\
\hline $20-24$ & $4(3.9 \%)$ & $0(0 \%)$ & $4(3.4 \%)$ \\
\hline $25-29$ & $7(6.8 \%)$ & $0(0 \%)$ & $7(6.0 \%)$ \\
\hline $30-34$ & $9(8.7 \%)$ & $0(0 \%)$ & $9(7.8 \%)$ \\
\hline $35-39$ & $19(18.4 \%)$ & $0(0 \%)$ & $19(16.4 \%)$ \\
\hline $40-44$ & $16(15.5 \%)$ & $1(7.7 \%)$ & $17(14.7 \%)$ \\
\hline $45-49$ & $15(14.6 \%)$ & $2(15.4 \%)$ & $17(14.7 \%)$ \\
\hline $50+$ & $33(32.0 \%)$ & $10(76.9 \%)$ & $43(37.1 \%)$ \\
\hline Total & $103(100 \%)$ & $13(100 \%)$ & $116(100 \%)$ \\
\hline
\end{tabular}

The other sociodemographic information that is documented on patients' records was their residence. Unfortunately, it couldn't be assessed for all patients as it was available only for $41.4 \%$ of all. However, from the 41 patients whose addresses could be retrieved $56.1 \%$ were found to be rural parts while the rest $43.9 \%$ came from urban areas.

\subsection{Clinical presentation and Risk factor assessment}

The most frequent chief complaint at presentation of patients was found to be breast lump. This accounted for $97.9 \%$ out of those whose clinical data was complete. In addition to breast lump, some patients had contemporary presenting symptoms of breast pain $(31.2 \%)$, nipple discharge $(12.5 \%)$, skin change or irritation $(10.5 \%)$ and nipple retraction $(2.1 \%)$.

The median duration of presenting symptom was found to be $8.5 \pm 14$ months for the 40 patients with available data. Fortunately, majority $(32.5 \%)$ had come after duration of illness of less than 6 months. However, those who came after 24 months and above have also contributed for significant figure which constituted $25 \%$ of the total. There were also patients who came as late as 60 months (5 years) after the presenting symptom.

Table 3: Frequency of the durations of illness of patients with breast cancer who are electively operated at JUSH from September 11,2010 to September 10, 2014 GC

\begin{tabular}{|l|l|l|}
\hline Duration of illness & Frequency & Percentage (\%) \\
\hline$<6$ months & 13 & 32.5 \\
\hline $6-11$ months & 8 & 20.0 \\
\hline $12-17$ months & 9 & 22.5 \\
\hline $18-23$ months & 0 & 0 \\
\hline $24+$ months & 10 & 25.0 \\
\hline Total & 40 & 100 \\
\hline
\end{tabular}

Out of the 103 female patients, the parity status of only 31 could be retrieved $(30.1 \%)$. From these majority $(80.6 \%)$ were found to be multiparous while only $19.4 \%$ were found to be nulliparous. The median parity was found to be $4 \pm 2$. But, parity of as high as Para X was reported.

Table 4: Parity status of patients with breast cancer who are electively operated at JUSH from September 11, 2010 to September 10,2014 GC

\begin{tabular}{|l|l|l|l|}
\hline \multicolumn{2}{|l|}{ Parity status } & Frequency & Percentage (\%) \\
\hline Nulliparous & 6 & 19.4 \\
\hline \multirow{4}{*}{ Multiparous } & Para I to II & 5 & 16.1 \\
\cline { 2 - 4 } & Para III to IV & 9 & 29.0 \\
\cline { 2 - 4 } & Para V to VI & 7 & 22.6 \\
\cline { 2 - 4 } & Para VII and above & 4 & 12.9 \\
\hline Total & 31 & 100 \\
\hline
\end{tabular}

Age at first child birth could be retrieved for few parous female patients. Of the eight who gave information on age at first child birth, all were found to be younger than 24 years during that time. Majority were also found to be even younger than 20 years at first child birth.

Not many of the parous women's' report on breast feeding habit could be retrieved. But of those with available data all have breast fed their children and the median duration was found to be $24 \pm 10.5$ months.
Age at menarche and age at menopause were one of the most poorly documented risk factors mentioned on patients' records. The age at menarche was reported for $9.7 \%$ of all female patients and the median age was found to be $15 \pm 1.3$ years. Age at menopause was reported for only $4.9 \%$. Fortypercent of those with the report entered menopause at age above 50 years.

Low response rates were also observed for the questions that inquired the rest of risk factors which included contraceptive use, history of smoking, 
alcohol abuse, previous breast lesion, prior familial history of breast cancer or any other related cancer. Contraceptive use was assessed in $21.35 \%$ of the female patients and only $36.4 \%$ were found to have a positive history. Surprisingly, there were 2 women who reported history of previous breast lesions even though the response rate for the category was quite low. None of the patients reported positive history of smoking, alcohol use, or family history of related cancer.

Coming to the physical examination findings at presentation, palpable breast mass was reported in majority (99\%) of 108 cases. Only 16 patients $(14.8 \%)$ had nipple retraction. Clinical presentation with fungating and/or ulcerating lesion on $\mathrm{P} / \mathrm{E}$ was reported in 34 patents (31.5\%). About 64 (59.8\%) had locally extensive lesion which included either extensions to skin (other than ulceration/fungation) or extension to underlying chest wall muscles. Breast lesion suspicious of inflammatory carcinoma was seen in only one patient $(0.9 \%)$.

There were 106 patients who had measurable breast mass on P/E at presentation. Majority of those had breast mass sizes of $2-5 \mathrm{~cm}(49.1 \%)$. Those who had mass size of $5 \mathrm{~cm}$ and above were also found to be many $(44.3 \%)$. The consistency of the mass was mainly reported as 'firm' subject to the examiner $(57.9 \%)$.

Table 5: Frequency of mass size at presentation among patients with breast cancer who are electively operated at JUSH from September 11,2010 to September 10, $2014 \mathrm{GC}$

\begin{tabular}{|l|l|l|}
\hline Mass size & Frequency & Percentage (\%) \\
\hline$<2 \mathrm{~cm}$ & 7 & 6.6 \\
\hline $2-5 \mathrm{~cm}$ & 52 & 49.1 \\
\hline$>=5 \mathrm{~cm}$ & 47 & 44.3 \\
\hline Total & 106 & 100 \\
\hline
\end{tabular}

Clinical presentation with axillary lymphadenopathy was reported in 98 patients $(90.7 \%)$. Out of those, $46.9 \%$ had fixed ipsilateral axillary lymphadenopathy. Supraclavicular lymphadenopathy was present in only 5 patients $(4.6 \%)$.
Distant metastasis was present in 13 out of 108 patients $(12 \%)$. The commonest mentioned site of distant metastasis was lung $(53.8 \%)$ followed by liver $(23.1 \%)$.

Table 6: Frequency of cancer with distant metastasis at time of presentation among patients with breast cancer who are electively operated at JUSH from September 11, 2010 to September 10, 2014 GC

\begin{tabular}{|l|l|l|l|}
\hline Distant metastasis & Frequency & Percentage (\%) \\
\hline \multirow{4}{*}{ Present } & Lung & 7 & 6.5 \\
\cline { 2 - 5 } & Liver & 3 & 2.8 \\
\cline { 2 - 5 } & Brain & 0 & 0 \\
\cline { 2 - 5 } & Bone & 0 & 0 \\
\cline { 2 - 5 } & Multiple & 3 & 2.8 \\
\hline Absent & 95 & 87.9 \\
\hline Total & 108 & 100 \\
\hline
\end{tabular}

Bilateral breast cancer was documented in two female patients who presented with locally advanced breast cancer of both sides at ages of 30 and 65 . But relevant familial breast cancer history was not found.
According to TNM clinical staging of breast cancer, majority of patients were found to have stage III disease at diagnosis $(64.8 \%)$. Those who presented with stage IV disease constituted $12 \%$. Stage I disease was seen in only 2 patients $(1.9 \%)$.

Table 7: TNM stage of cancer at time of presentation among patients with breast cancer who are electively operated at JUSH from September 11, 2010 to September 10,2014 GC

\begin{tabular}{|l|l|l|}
\hline TNM stage & Frequency & Percentage $(\%)$ \\
\hline Stage I & 2 & 1.9 \\
\hline Stage II & 23 & 21.3 \\
\hline Stage III & 70 & 64.8 \\
\hline Stage IV & 14 & 12.0 \\
\hline Total & 108 & 100 \\
\hline
\end{tabular}

\subsection{Pathology diagnosis and Modes of treatment delivered}

Pathology reports could be retrieved only for $29.3 \%$ of patients. From these, Ductal invasive carcinoma was found to be the commonest histological type (79.4\%). Histological grade was documented for few patients $(8.6 \%)$. And high grade histology was seen in $30 \%$ of those. 
Regarding the mode of surgical treatment, in the majority of patients MRM with axillary dissection was done accounting for $83.6 \%$. Toilet mastectomy was the next frequently done procedure $(8.6 \%)$ mainly for patients with Stage IV cancers. BCT with axillary dissection was done only in a single patient who had a Stage II disease. In addition to MRM and axillary dissection, breast reconstruction was attempted with trapezius flap in one patient.

Table 8: Frequency of different modes of surgical treatments provided for patients with breast cancer who are electively operated at JUSH from September 11, 2010 to September 10, 2014 GC

\begin{tabular}{|l|l|l|}
\hline Mode of surgical treatment & Frequency & Percentage (\%) \\
\hline MRM \& axillary LN dissection & 97 & 83.6 \\
\hline Toilet mastectomy & 10 & 8.6 \\
\hline Excisional biopsy & 3 & 2.6 \\
\hline Simple mastectomy & 2 & 1.7 \\
\hline BCT \& axillary LN dissection & 1 & 0.9 \\
\hline MRM \& Breast reconstruction & 1 & 0.9 \\
\hline Other & 2 & 1.7 \\
\hline Total & 116 & 100 \\
\hline
\end{tabular}

Post-operative complications were seen in 14 patients $(13 \%)$. The most frequent complication seen was flap necrosis which was seen in 6 patients $(5.2 \%)$. Other mentioned complications included seroma formation $(3.4 \%)$, wound infection $(1.7 \%)$ and anesthesia related complications $(2.6 \%)$.

The genuine figure of the continuation of adjuvant treatment was difficult to retrieve. However, 17 out of the total number of patients have received some kind of adjuvant treatment $(14.7 \%)$. There were 11 female patients who had bilateral Oophorectomy done during or after the definitive surgery $(9.5 \%)$. The rest were having records of anti-estrogen treatment in their follow-up charts $(5.2 \%)$. Only one patient was documented to have chemotherapy after the surgery. There was no patient who was reported to have radiotherapy.

Very few patients showed-up for check-up after discharge $(10.3 \%)$. Some of who didn't show had referral notes documented which makes their failure to come for follow-up convincing. But still others have poor documentations, thus making it difficult to draw any conclusions. The longest documented period of follow-up after discharge was 19 months and the median duration was found to be 6 months.

Post-operative recurrence was documented in only seven patients $(6 \%)$. Commonly, these patients had local recurrence $(62.5 \%)$. In addition there were patients who were found to have distant and axillary recurrences. Re-operations were done for those patients who had local recurrence except one. However, it is difficult to conclude that the rest of patients didn't have recurrence at all as few have presented for follow-up after discharge.

\section{Discussion}

This study is very similar in its nature to the retrospective study done at Tikur Anbessa Hospital from 1995-1999 GC (14). However the review done at JUSH is for a period of four years duration while that of the former is for a period of five years. This has been due to the inconvenient discarding of charts from the card room that have stayed 5 years and longer. Even though it is difficult to make exact comparisons between the two studies, one can estimate the similarities and differences on breast cancer trends observed between the populations the two hospitals serve.

The study done at TAH by Professor Tesema was based on a total of 137 biopsy proven breast cancer cases of which 125's data could be retrieved and analyzed. The study tried to assess sociodemographic information, clinical presentation, TNM staging and Pathology diagnosis and modes of treatment delivered and outcomes for breast cancer cases in the hospital setup (14).

In the presence of some limitations, the current study has also tried to assess all the above mentioned factors. In addition, it has also aimed to assess risk factors of breast cancer seen in the setup despite the poor record of data that could be retrieved. In total, 116 patients who were also having biopsy proven diagnosis of breast cancer were recruited for the study based on the information obtained from the operation room log book. Out of the total 116 cases, the clinical data could be retrieved for 108 cases. But still complete records were barely available making it difficult to withdraw genuine conclusions from some parts such as clinical presentation and risk assessment of the breast cancer patients.

The sociodemographic review of the current study has revealed that the male to female sex ratio to be $1: 8$. This figure is similar to the result revealed by the study done in TAH (14). The rate of male breast cancer was found to be $11.2 \%$ in this study which is roughly close to the figure $(10.4 \%)$ seen in TAH (14). This shows that in the Ethiopian population the rate of male breast cancer is higher than the world's 
pattern which is known to be less than $1 \%$ (3). In text books it is generally mentioned that male breast cancer is rare among the Western population. In the Western world, incidence is reported to be highest among North Americans \& British in whom breast cancer constitutes $1.5 \%$ of all male cancers (3). Jewish and African Americans are also known to have highest incidence of male breast cancer (3). Because of the lack of adequate cancer registry system in our country, we cannot point what the breast cancer constitutes among male population of Ethiopia. But from the current study and the study done in TAH, one can see male breast cancer is not rare but rather one of the common encounters in surgical practice. Large scale studies in the country may need to be done to know the exact magnitude of the problem.

The other attention seeking fact revealed within the sociodemographic review is the age distribution of breast cancer patients, more importantly of females. Although the age of female patients ranged from 20 to 82 years, majority of the women were younger than 50 years $(68 \%)$. And those who were younger than 30 years constituted $10.7 \%$. The mean age for females was found to be $43.72 \pm 12.8$. The pioneer research done in TAH has revealed similar facts that majority of Ethiopian women with breast cancer are pre-menopausal and more than half are having age less than 50 years (14). It has also mentioned that women who were younger than 30 years contributed for $13.4 \%$ while those who were younger than 50 contributed for $70.5 \%$ (14). This disparity from the world's epidemiological trend of breast cancer is also seen in other African countries. The study done on Sudanese women revealed that $70 \%$ of the women were younger than 50 years (11). Another study done in Mali has shown the median age for women with breast cancer to be 46 years (10). Similar findings are also reflected in other developing countries. A systematic review of breast cancer biology in developing countries has tried to assess the trend in different continents and has concluded that early age of onset is observed in many of the countries (13).

The consistently younger age of onset of breast cancer among African women has already drawn the attention of world researchers. The consistent decline in breast cancer risk after menopause in different regions of Africa is in sharp contrast to the rising incidence rates seen in post-menopausal women of North America and Europe (2). One of the studies comparing breast cancer in African-American women with that of Sub-Saharan Africa has reported breast cancer incidence peaks between ages 35-45 in Africa which is approximately 10-15 years earlier than the peak incidence for Western countries (2). This study has attributed this disparity to different causes. One of the arguments it focuses is the low life expectancy of the African population. It says that the unfortunate truncation of life expectancy precludes the ability to make robust conclusions regarding the risk of postmenopausal cancer (2). In addition the high illiteracy rate, poor socioeconomic status, and decreased breast cancer awareness among older African women makes the case ascertainment disproportionately low in this group (2).

The ethnic group of patients and their religion was not assessed in the current study since the information is not available on patients' charts. Had it been known, the differences in incidence among different groups would have reflected the background on genetics of breast cancer among the Ethiopian population. The study done by Prof Tesema has revealed that majority of cases seen were Amhara $(63.5 \%)$ by ethnicity and Christians $(86.4 \%)$ by religion (14).

Parity is one of the important gynecologic and reproductive factors when talking about breast cancer risks. It is well known that multiparity is a protective factor while nulliparity is a risk to breast cancer as this has an effect on the number of ovulatory cycles a woman gets exposed to in life time. This dogmatic understanding we have on breast cancer is challenged when it comes to trends seen in the African setup. Studies have documented increased parity in African communities averaging 5-9 live births per woman (2). The study done in TAH has reported median parity of $4 \pm 2$ while nulliparous women constituted $18.1 \%$. Although the number of respondents on parity status has been low in the current study, similar figures were found where median parity was $4 \pm 2$ and nulliparity was seen in only $19.4 \%$ of women. However, in the Ethiopian community where marriage and child birth has high regard in a life of a woman, these figures wouldn't be surprising, especially when remembering that the higher percentage of cases in this study have come from rural area. Although high parity together with other reproductive factors contributes for the relatively lower cumulative incidence of breast cancer in African countries like Ethiopia, the casual relationship between parity as a risk and breast cancer needs further elaboration.

In addition to parity, the other hormone related protective factors to breast cancer in a woman's reproductive life have been reviewed in this study. Fortunately, most women were found to have protective factors. For instance, it is worthwhile to mention that breast feeding was practiced by all parous women whose response could be retrieved and that majority lactated for a prolonged time the median duration being $24 \pm 10$ months. Additionally, all women whose age at first child birth was mentioned started child bearing when they were 
younger than 25 years. The median age at first child birth was found to be $19 \pm 2.4$.Menarche started to be experienced by most late with median age $15 \pm 1.3$ years. These go together with the findings of studies done in most African countries, where late menarche (median, 15 years), early child bearing with median age of 19, prolonged breast feeding where $96 \%$ breast fed for average of 16 months, has been reported (2). There were also studies that questioned the relation of these hormone related factors to breast cancer risk like the study done in Nigeria which assessed and found no correlation of breast cancer to age at menarche, to parity and to prolonged lactation in about 2000 Africans (2). Thus, researching the strength of those hormone related risk factors effect on the incidence of breast cancer in the Ethiopian context will remain an assignment.

On the other hand, the incidence of breast cancer in Africa is rising which is explained to be due to the adoption of western life style especially by the welleducated, well-to-do and urbanized community. In Uganda, the incidence of breast cancer has doubled from 11 per 100,000 in 1961 to 22 per 100,000 in 1995 GC (2). In South Africa, a study has reported doubling of incidence rates in women living in urban areas when compared to those living in rural parts (2). This has been stated in the world's GLOBOCAN report of 2012 by IARC which emphasized that breast cancer, a number one killing cancer among women, is seen to increase in incidence in the less developed countries due to the rapid economic and societal changes leading to shift in life style typical of the industrialized world (7). Potential risk factors such as obesity, diet, socioeconomic status, and level of education and their effect on the incidence of breast cancer have not been assessed by the current or other studies done in Ethiopia. The author would like to emphasize the undeniable importance of knowing the effect of shift of life style on breast cancer as Ethiopia is known now days to be one of the countries with fast growing economies.

Early detection of breast cancer, its diagnosis and treatment is one of the recommendations suggested by IARC to the less developed countries of the world for a rising burden of breast cancer morbidity and mortality is seen in these countries (7). Late presentation of breast cancer patients in less developed countries like Ethiopia leads to presentations with advanced cancers that are beyond curability and to overall poor prognosis of patients. In the current study, the median duration of illness at presentation was found to be $8.5 \pm 14$ months. However, those who came after being sick for 24 months and above constituted a significant percent (25\%) confirming late presentation of patients. The study done in TAH showed even a longer duration of illness at presentation (11.5 \pm 19.3 months) (14). Even though it is believed that the health seeking behavior of the rural community of Ethiopia has grown through the enormous work done by $\mathrm{MOH}$, this has not been well proven in aspects of noncommunicable conditions such as cancer. One study done in Ethiopia found that early signs and symptoms of breast cancer were ignored frequently and that patients preferred to go to traditional healers (4). In addition, it says that most patients experience stigmatization and social isolation which complicated discussion and action around breast cancer (4). Another study also done in Ethiopia reported that most breast cancer patients (69.6\%) ignored symptoms for average of more than one and half years (15). In summary, all the mentioned studies point that breast cancer patients present late despite noticing of initial symptoms, which is found to be commonly breast lump in the current $(97.9 \%)$ and other studies, mainly due to cultural and religious beliefs related with stigmatization and social isolation.

The magnitude of the problem of late presentation is further magnified when we see the stages of disease patients had at initial presentation in the current study and other similar studies. Advanced stage of breast cancer (Stage III \&IV) constituted $76.8 \%$ of the total. This figure is even higher than the condition reported in TAH which was $60.2 \%$ (14). Additionally, $31.5 \%$ of patients in the current study presented with ulcerated and fungated lesions. The corresponding figure in the TAH study was found to be $25 \%$. Presentation of breast cancer patients with advanced stage is also manifested in other African countries. In Sudan, majority came with Stage III \& IV disease (60.7\%) (11). In Mali, T3 - T4 disease was seen in $90 \%$ of cases $(10 \%)$. In Tanzania, again, $76 \%$ had stage IIIB disease at presentation and none were found Stage I disease (13). Another study reviewing publications in Africa has shown 70-90\% of African women present with stage III to IV disease (2).

Distant metastasis of breast cancer at presentation per se contributed for significant burden. In the current study, $12 \%$ had distant metastasis with the most frequent site being the lung. Similarly, $10 \%$ of patients in TAH were reported to have distant metastasis at presentation (14). Identical reports have been made from Tanzania (13).

Another measure for late presentation of patients with breast cancer is the lymph node status at presentation. In the current study, $90.7 \%$ had axillary LAP at presentation while $48.1 \%$ had fixed lymph nodes. This finding is even worse than the situation seen in other studies. In TAH, 54\% had mobile axillary LAP and $16 \%$ had fixed lymph nodes (14). In Sudan, greater than half had nodal involvement at presentation (11). In Mali, positive lymph nodes were 
seen in $91 \%$ of cases (10). The degree of nodal involvement at presentation is an indirect way to measure late presentation of patients magnifying the rarity of finding early stage disease in the African setup.

In addition to lack of awareness, social factors and problems of health facility affecting the late presentation of patients, the aggressive pathological characteristics of breast cancer cases seen in African women partly contributes for the problem. This is manifested by fast progression of disease within short period of time (2). Findings of Hassen et al support this theory; they found that 18 out of 21 patients $(81 \%)$ with a brief symptom duration (3 months or less) had stage III or IV disease (2). Concerning the histological type, we know from the current study and other studies that it largely (85\% in average) consists of Ductal invasive carcinoma $(14,11,10,2)$. High grades of tumor have also been reported (10). Additionally, triple negativity is uniformly seen in most of the studies done in Africa, precluding Ethiopia where receptor positivity and HER-2 neu status is not being determined so far. For instance, in Mali majority of the cancers were found to be estrogen and progesterone receptor negative $(61 \%$ \& $72 \%$ respectively) and Her-2 was over expressed in $18 \%$ of cases (10). In total triple negativity was found to be $46 \%$ (10). Her-2/neu overexpression per se has been associated with advanced tumor grade, size, nodal status, and with shorter disease-free and overall survival especially in patients with node-positive tumors (2). Its role in node negative breast cancers has been more controversial (2). Even though the detailed biology and genetics of breast cancer is not studied in Ethiopia, it may be true that aggressive type of breast cancer is seen among patients and this partly contributes for the presentation of patients with advanced disease.

The building of infrastructure to improve access to health and focused attempts to increase the number of fully equipped cancer centers in the country will undoubtedly have an impact in the future in improving early detection \& treatment of breast cancer. But preliminary works such as working on the awareness of both illiterate and literate women towards breast cancer, teaching of breast selfexamination techniques, and organizing breast cancer screening programs will definitely have tremendous impact in combating breast cancer in Ethiopia. This will remain an invaluable assignment for clinicians, public health workers and policy makers. Additionally, as one of the second common cancer in the country, next to cervical cancer, more budget needs to be allocated for researching on the biological aggressiveness and genetic tendency of breast cancer.
Regarding the treatment of breast cancer, surgical treatment is adequately delivered for those who have made it on time to proper health facilities. In the current study, MRM and axillary lymph node dissection is commonly performed $(83.6 \%)$. But the deficiency in treatment lies in access to the wing of adjuvant therapies. In our case, only $14.7 \%$ had some kind of adjuvant treatment. Due to the unaffordability of referral to the only cancer treatment center available in the country, it was observed that most surgeons empirically either start Tamoxifen or perform bilateral Oophorectomy. There are promising things being seen as JUSH is in the movement of building an oncologic center in the hospital and also training specialists on the field. But still urgent attention to the problem and prompt solutions should be sought to salvage of the life of the economically unprivileged breast cancer patients that come largely from rural areas.

\section{Conclusion}

- This study in harmony with another study has shown that in the Ethiopian population the rate of male breast cancer is higher than the world's pattern which is known to be less than $1 \%$

- In contrast to the epidemiological trends of breast cancer in the world, majority of breast cancer patients were found to be younger than 50 years.

- Despite having protective gynecologic and reproductive factors, Ethiopian women are seen to be affected with breast cancer. The findings which were found positive in this study include high parity, early start of child bearing, prolonged lactation and late menopause.

- Other risk factors of breast cancer related with shift to western life style have not been assessed in this and other studies done in the country. But the association of obesity, diet, socioeconomic status and educational level with breast cancer may have impacted the rising incidence of breast cancer in Ethiopia.

- Breast cancer patients in this study were found to have late presentations after ignoring early signs and symptoms like breast lump. They were also found to have advanced stage of disease and high percentage of nodal involvement at the time of initial presentation.

- Complete biological and pathological analysis of breast cancer cases couldn't be done in the current study since the information is not available. But this leaves an information gap whether presentation of patients with advanced disease within short duration of illness can be attributed to aggressive characteristics of the tumor.

- The percentage of patients who received adjuvant treatment as part of the comprehensive 
treatment of breast cancer was found to be very low in this study.

\section{Recommendations}

- A prospective study on breast cancer should be done at JUSH to more elaborately understand the epidemiological trends and risk factors of patients that come from within the catchment area of the hospital so that possible deviations from the international trends would be known and acted upon.

- Clinicians, public health workers and policy makers should act on increasing the awareness of the Ethiopian population towards breast cancer by using health informatics about the disease, teaching techniques of breast self-examination, launching of screening programs so that patients with breast cancer would seek attention of a health worker in the presence of early signs and symptoms.

- Budget should be allocated by teaching university hospitals and other concerned bodies so that further biological and genetical characterization of breast cancer would be done in the Ethiopian context starting from the preliminary determination of receptor positivity Her-2/neu status.

- JUSH should work on ways to facilitate comprehensive care of all breast cancer patients including availability of adjuvant treatment to improve survival of patients.

\section{References}

1. Anim JT. Breast cancer in sub-Saharan African women. Afr J Med Med Sci. 1993 Mar;22(1):5-10.

2. Alero Fregene \& Lisa A. Newman. Breast Cancer in Sub-Saharan Africa: How Does it Relate to Breast
Cancer in African-American Women? CANCER (2005); 103(8): 1540-1550

3. Brunicardi F Charles and others. Schwartz's Principles of Surgery, $9^{\text {th }}$ ed.2010

4. De Ver Dye T and others. A mixed-method assessment of beliefs and practice around breast cancer in Ethiopia: Implication for public health programming and cancer control. Global Public Health: An International Journal for Research, Policy \& Practice (2011); 6(7):719-31

5. International Agency for Cancer Research. GLOBOCAN 2012: Estimated Cancer incidence, Mortality and Prevalence Worldwide in 2008.

6. International Agency for Cancer Research GLOBOCAN 2012: Estimated Cancer incidence, Mortality and Prevalence Worldwide in 2012.

7. International Agency for Cancer Research (IARC), No 223, 2013

8. John P.A., Moses G., Olivia K. and Jane O.F. High serum Estradiol confers no risk for breast cancer: another disparity for Sub-Saharan African women. Pan African Medical Journal (2012); 12: 23

9. Jonathan S. Berek. Berek \&Novak's Gynecology, $14^{\text {th }}$ ed. 2007

10. Madani Ly and others. High Incidence of tripleNegative Tumors in Sub-Saharan Africa: A Prospective Study of Breast Cancer Characteristics and Risk Factors in Malian Women in Bamako University Hospital. Oncology (2012); 83:257-263

11. Omer EL Faroug H Salim. Breast Cancer in Africa; Are we dealing with a different disease. Sudan Medical Journal (2014); 50(1)

12. Ries LAG and others. SEER Survival Monograph: Cancer Survival Among Adults: U.S. SEER Program, 1988-2001. National Cancer institute, SEER Program, NIC Pub. No. 07-6215, Bethseda, MD, 2007.

13. Riyaz Bhikoo and others. Systematic Review of Breast Cancer Biology in Developing Countries (Part 1): Africa, the Middle East, Eastern Europe, Mexico, the Caribbean and South America. CANCER (2011);3,2358-2381

14. Tessema Ersumo. Breast Cancer in an Ethiopian Population, Addis Ababa. East and Central African Journal of Surgery (2006); 11 (1): 81-86

15. Timothy D. Dye \& Solomon Bogale. Experience of initial symptoms of breast cancer and triggers for action in Ethiopia. International Journal of Breast Cancer (2012);908547:5

\section{ANNEX-I \\ QUESTIONNAIRE}

Questionnaire on retrospective analysis of breast cancer cases operated at JUSH from September 11, 2010 to September 10, 2014 GC.

Part I: Socio-demographic information
SD1. Age: years
SD2. Sex:
1. Female
1. Rural
2. Male
2. Urban

SD3. Residence: 1 . Rural

Part II: Clinical presentation and Risk assessment

CP1. What was the presenting complaint? (More than one can be checked)

\begin{tabular}{|l|l|l|l|}
\hline Code & Presenting Complaint & Yes & No \\
\hline CP1.1 & Breast lump & 1 & 0 \\
\hline CP1.2 & Breast pain & 1 & 0 \\
\hline CP1.3 & Nipple retraction & 1 & 0 \\
\hline CP1.4 & Skin change or irritation & 1 & 0 \\
\hline CP1.5 & Reddness or Scaliness of nipple/areola & 1 & 0 \\
\hline CP1.6 & Breast swelling (whole or part of it) & 1 & 0 \\
\hline CP1.7 & Nipple discharge & 1 & 0 \\
\hline CP1.8 & Other & 1 & 0 \\
\hline
\end{tabular}

CP2. Duration of illness: months

CP3. What was the parity of the patient? (Applies for female patients only)

$$
\text { 1. Nulliparous }
$$

CP4. Specify the parity of parous female patients: 
CP5. Mention the age at first child birth for parous female patients: years

CP6. Was there history of breast feeding? (Applies for parous female patients only) $\begin{array}{ll}\text { 1. No } & \text { 2. Yes }\end{array}$

CP7. Mention the duration of breast feeding for those who had the history: ____ months

CP8. Mention patient's age at menarche:___ years

CP9. Mention patient's age at menopause for postmenopausal patients: years

\begin{tabular}{|l|l|l|l|}
\hline Code & Question & Yes & No \\
\hline CP10. & History of contraceptive use & 1 & 0 \\
\hline CP11. & Smoking history & 1 & 0 \\
\hline CP12. & Alcohol use & 1 & 0 \\
\hline CP13. & Previous breast lesion & 1 & 0 \\
\hline CP14. & $\begin{array}{l}\text { History of breast and/or ovarian cancer } \\
\text { among first degree relatives }\end{array}$ & 1 & 0 \\
\hline CP15. & Family history of any other cancer & 1 & 0 \\
\hline
\end{tabular}

CP16. Description of breast lesion:

\begin{tabular}{|l|l|l|l|}
\hline Code & Question & Yes & No \\
\hline CP16.1. & Was mass present? & 1 & 0 \\
\hline CP16.2. & Was nipple retracted? & 1 & 0 \\
\hline CP16.3. & Was mass tender? & 1 & 0 \\
\hline CP16.4. & $\begin{array}{l}\text { Was mass fixed to } \\
\text { surrounding structures? }\end{array}$ & 1 & 0 \\
\hline CP16.5. & $\begin{array}{l}\text { Were fungating lesion and/or } \\
\text { ulceration present? }\end{array}$ & 1 & 0 \\
\hline CP16.6. & $\begin{array}{l}\text { Was extension to kin other } \\
\text { than ulceration present? }\end{array}$ & 1 & 0 \\
\hline CP16.7. & $\begin{array}{l}\text { Was extension to chest was } \\
\text { muscles present? }\end{array}$ & 1 & 0 \\
\hline CP16.8. & $\begin{array}{l}\text { Was there suspicion of } \\
\text { inflammatory carcinoma? }\end{array}$ & 1 & 0 \\
\hline
\end{tabular}

CP16.9. What was the mentioned mass size?

$1 . \quad$ Less than $2 \mathrm{~cm}$
CP16.10. What was the consistency of the mass?

2. $2-5 \mathrm{~cm} 3$. Greater or equal to $5 \mathrm{~cm}$
1. Soft
2. Firm
3. Hard

\begin{tabular}{|c|l|l|l|}
\hline Code & Question & Yes & No \\
\hline CP17. & $\begin{array}{l}\text { Was ipsilateral axillary lymphadenopathy } \\
\text { present? }\end{array}$ & 1 & 0 \\
\hline CP18. & Was axillary lymphadenopathy fixed? & 1 & 0 \\
\hline CP19. & Was supraclavicular lymphadenopathy present? & 1 & 0 \\
\hline CP20. & Was distant metastasis present? & 1 & 0 \\
\hline
\end{tabular}

CP21. If distant metastasis was present specify the site:

CP22. What was the TNM Stage of disease:
1. Stage I
2.Stage II 3. Stage III4. Stage IV

Part III: Pathology Diagnosis and Treatment Given

PT1. FNAC/ Biopsy Diagnosis:

PT2. What was mode of definitive surgical treatment provided? (more than one can be checked)

\begin{tabular}{|l|l|l|l|}
\hline Code & Surgical Treatment & Yes & No \\
\hline PT2.1 & MRM & 1 & 0 \\
\hline PT2.2 & Simple mastectomy & 1 & 0 \\
\hline PT2.3 & BCT & 1 & 0 \\
\hline PT2.4 & Excisional biopsy & 1 & 0 \\
\hline PT2.5 & Toilet mastectomy & 1 & 0 \\
\hline PT2.6 & Axillary LN dissection & 1 & 0 \\
\hline PT2.7 & Other & 1 & 0 \\
\hline
\end{tabular}

PT3. Was post-operative complications seen after surgery?
1. No
2. Yes

PT4. If complications were seen after the operation specify: (more than one can be checked)

\begin{tabular}{|l|l|l|l|}
\hline Code & Post-op complication & Yes & No \\
\hline PT4.1 & Wound infection & 1 & 0 \\
\hline PT4.2 & Flap necrosis & 1 & 0 \\
\hline PT4.3 & Lymphedema & 1 & 0 \\
\hline PT4.4 & VTE & 1 & 0 \\
\hline PT4.5 & Seroma & 1 & 0 \\
\hline PT4.6 & Hematoma & 1 & 0 \\
\hline PT4.7 & Other & 1 & 0 \\
\hline
\end{tabular}


PT5. Patient showed up for follow-up after discharge:

$$
\begin{array}{ll}
\text { 1. No } & \text { 2. Yes }
\end{array}
$$

PT6. Mention the duration of follow-up after discharge if patient ever showed up: months

PT7. Was there any documented recurrence?

1. No 2.Yes

PT8. What was the type if recurrence was documented?
1. Local

PT9. Was re-operation done for a mentioned local recurrence?

$$
\begin{array}{ll}
\text { 1. No } 2 \text {. Yes } & \\
&
\end{array}
$$

PT10. Was any adjuvant modality of treatment delivered?

$$
\begin{array}{ll}
\text { 1. No } & \text { 2. Yes }
\end{array}
$$

PT11. If adjuvant treatment was delivered what was the type?
1. Radiation
2. Chemotherapy
3. Anti- estrogen therapy 4. Ablative endocrine therapy (Bilateral Oopherectomy)

\begin{tabular}{|c|c|}
\hline Primary Tumor $(\mathbf{T})$ & Definitions \\
\hline $\mathrm{Tx}$ & Primary tumor cannot be assessed \\
\hline T0 & No evidence of primary tumor \\
\hline Tis & Carcinoma in situ \\
\hline $\mathrm{T} 1$ & Tumor $\leq 2 \mathrm{~cm}$ in greatest dimension \\
\hline $\mathrm{T} 2$ & Tumor $>2 \mathrm{~cm}$ but not $>5 \mathrm{~cm}$ in greatest dimension \\
\hline T3 & Tumor $>5 \mathrm{~cm}$ in greatest dimension \\
\hline T4 & Tumor of any size with direct extension to (a) chest wall or (b) skin, only as described below \\
\hline T4a & Extension to chest wall, not including pectoralis muscle \\
\hline $\mathrm{T} 4 \mathrm{~b}$ & $\begin{array}{l}\text { Edema (including peau d'orange), or ulceration of the skin of the breast, or satellite skin } \\
\text { nodules confined to the same breast }\end{array}$ \\
\hline $\mathrm{T} 4 \mathrm{c}$ & Both T4a and T4b \\
\hline $\mathrm{T} 4 \mathrm{~d}$ & Inflammatory carcinoma \\
\hline Regional Lymph Nodes- Clinical (N) & Definitions \\
\hline $\mathrm{Nx}$ & Regional lymph nodes cannot be assessed (e.g., previously removed) \\
\hline N0 & No regional lymph node metastasis \\
\hline $\mathrm{N} 1$ & Metastasis to movable ipsilateral axillary lymph node(s) \\
\hline $\mathrm{N} 2$ & $\begin{array}{l}\text { Metastases in ipsilateral axillary lymph nodes fixed or matted, or in clinically apparent } \\
\text { ipsilateral internal mammary nodes in the absence of clinically evident axillary lymph node } \\
\text { metastasis }\end{array}$ \\
\hline $\mathrm{N} 2 \mathrm{a}$ & $\begin{array}{l}\text { Metastasis in ipsilateral axillary lymph nodes fixed to one another (matted) or to other } \\
\text { structures }\end{array}$ \\
\hline N3 & $\begin{array}{l}\text { Metastasis only in clinically apparent ipsilateral internal mammary nodes and in the absence of } \\
\text { clinically evident axillary lymph node metastasis; metastasis in ipsilateral infraclavicular } \\
\text { lymph node(s) with or without axillary lymph node involvement, or in clinically apparent } \\
\text { ipsilateral internal mammary lymph node(s) and in the presence of clinically evident axillary } \\
\text { lymph node metastasis; or metastasis in ipsilateral supraclavicular lymph node(s) with or } \\
\text { without axillary or internal mammary lymph node involvement }\end{array}$ \\
\hline N3a & Metastasis in ipsilateral infraclavicular lymph node(s) \\
\hline $\mathrm{N} 3 \mathrm{~b}$ & Metastasis in ipsilateral internal mammary lymph nodes(s) and axillary lymph node(s) \\
\hline $\mathrm{N} 3 \mathrm{c}$ & Metastasis in ipsilateral supraclavicular lymph node(s) \\
\hline Distant Metastasis (M) & Definitions \\
\hline Mx & Distant metastasis cannot be assessed \\
\hline M0 & No distant metastasis \\
\hline M1 & Distant metastasis \\
\hline
\end{tabular}

\section{ANNEX- II}

Tnm Clinical Staging System for Breast Cancer

TNM Stage Groupings

\begin{tabular}{|l|l|l|l|}
\hline Stage 0 & Tis & N0 & M0 \\
\hline Stage I & T1 & N0 & M0 \\
\hline Stage IIA & T0 & N1 & M0 \\
\hline & T1 & N1 & M0 \\
\hline & T2 & N0 & M0 \\
\hline Stage IIB & T2 & N1 & M0 \\
\hline & T3 & N0 & M0 \\
\hline Stage IIIA & T0 & N2 & M0 \\
\hline & T1 & N2 & M0 \\
\hline & T2 & N2 & M0 \\
\hline & T3 & N1 & M0 \\
\hline Stage IIIB & T3 & N2 & M0 \\
\hline
\end{tabular}


Retrospective Analysis of Breast Cancer Cases Operated in Jush within Four Years Time Period, Jimma, Ethiopia

\begin{tabular}{|l|l|l|l|}
\hline & T4 & N1 & M0 \\
\hline & T4 & N2 & M0 \\
\hline Stage IIIC & Any T & N3 & M0 \\
\hline Stage IV & Any T & Any N & M1 \\
\hline
\end{tabular}

Source: AJCC cancer staging manual, 6th ed. New York: Springer 2002, pp 228.

\section{Acronyms}

AJCC- American Joint Committee on Cancer

BCT- Breast Conserving Therapy

ER- Estrogen Receptor

HRT- Hormone Replacement Therapy

IARC- International Agency for Research on Cancer

JU- Jimma University

JUSH- Jimma University Specialized Hospital

LN- Lymph Node

LAP- Lymphadenopathy

MRM- Modified Radical Mastectomy

PR- Progesterone Receptor

TNM- primary Tumor, clinical Node and distant Metastasis 